\title{
Avaliação do desempenho das redes de atenção à saúde: uma proposta de indicadores
}

\author{
Evaluation of health care networks: a proposal for key performance \\ indicators
}

\section{Evaluación del desempeño de las redes de atención de salud: una propuesta de indicadores}

\author{
Daiane Ellwanger Araujo | daiane.earaujo@gmail.com \\ Universidade de Brasília. Brasília, DF, Brasil. \\ Edgar Merchan-Hamann | merchan.hamann@gmail.com \\ Universidade de Brasília. Brasília, DF, Brasil.
}

Francisca Sueli da Silva Lima | suelisabino carneiro@yahoo.com.br

Universidade de Brasília. Brasília, DF, Brasil.

Josué Laguardia |josue.laguardia@gmail.com

Fundação Oswaldo Cruz. Rio de Janeiro, RJ. Brasil.

Maria Margarita Urdaneta Gutierrez | urdanetamm@gmail.com

Universidade de Brasília. Brasília, DF, Brasil.

\section{Resumo}

Este artigo baseia-se em um estudo descritivo de processo metodológico de natureza operacional para identificar indicadores que permitam a avaliação do desempenho das Redes de Atenção à Saúde (RAS) mediante a identificação de agravo de saúde pública no contexto da regionalização do Sistema Único de Saúde. Os métodos de seleção do agravo e indicadores incluíram a realização de três oficinas com especialistas interdisciplinares aplicando a Técnica de Grupo Nominal. Priorizou-se o agravo câncer do colo do útero e chegou-se a uma composição final de 10 indicadores para avaliação de subdimensões do desempenho dos componentes da estrutura operacional da RAS. Concluiu-se que um conjunto de indicadores factíveis e validados para o agravo selecionado pode compor um sistema de monitoramento e avaliação das RAS de modo a subsidiar gestores na tomada de decisão. Os desdobramentos futuros do estudo em questão serão o cálculo e a apresentação dos indicadores para comparabilidade entre as regiões de saúde do Brasil, bem como a validação da metodologia proposta.

Palavras-chave: indicadores de saúde; avaliação de desempenho; Sistema Único de Saúde; sistemas de informação em saúde; regionalização. 


\begin{abstract}
This article is based on a descriptive study of applied nature to identify indicators for assessing performance on the network of health care (RAS) for public health problems selected under the context of the regionalization of the Brazilian Unified Health System (Sistema Único de Saúde). For selecting the disease and performance indicators, we held three workshops getting together interdisciplinary specialists and applying Nominal Group Technique. Specialists priorized uterine colon cancer and proposed ten indicators for evaluating the performance of sub-dimensions of the components of the operational structure of the RAS. We conclude that a set of feasible and validated performance indicators can compose a monitoring and evaluation system of RAS which can be used by health managers in the decision-making process. Future developments of this study are calculation and presentation of the indicators for comparison among health regions in Brazil and validation of the applied methodology.
\end{abstract}

Keywords: health indicators; appraisal of performance; Sistema Único de Saúde (Unified Health System); health information systems; regional health planning.

\title{
Resumen
}

Estudio descriptivo de carácter operacional para identificar los indicadores para evaluar el desempeño de las redes de atención de salud (RAS) para problemas de salud en el contexto de la regionalización del Sistema Único de Saúde (Sistema Único de Salud). Los métodos incluyeron la realización de tres talleres con especialistas de distintas áreas profesionales y aplicación de la Técnica de Grupo Nominal. Se priorizó el cáncer de cuello uterino y se llegó a una composición final de diez indicadores para evaluación del desempeño de las subdimensiones de los componentes de la estructura operativa de la RAS. Se concluye que un conjunto de indicadores de resultados factibles y validados puede constituir un sistema para supervisar y evaluar las RAS para subsidiar gerentes en la toma de decisiones. Futuros desarrollos de la investigación son el cálculo y la presentación de los indicadores para comparación entre las regiones de salud brasileñas y validación de la metodologia propuesta.

Palabras clave: indicadores de salud; evaluación del desempeño; Sistema Único de Saúde (Sistema Unico de Salud); sistemas de información de salud; regionalización.

INFORMAÇÕES DO ARTIGO

\begin{abstract}
Contribuição dos autores:
Concepção e desenho do estudo: Daiane Ellwanger Araujo, Francisca Sueli da Silva Lima e Maria Margarita Urdaneta Gutierrez. Aquisição, análise ou interpretação dos dados: Daiane Ellwanger Araujo, Francisca Sueli da Silva Lima e Maria Margarita Urdaneta Gutierrez. Redação do manuscrito: Daiane Ellwanger Araujo, Edgar Merchan-Hamann, Josué Laguardia e Maria Margarita Urdaneta Gutierrez. Revisão crítica do conteúdo intelectual: Edgar Merchan-Hamann e Josué Laguardia.
\end{abstract}

Declaração de conflito de interesses: Os autores declaram não existir conflito de interesse referente à elaboração do artigo.

Fontes de financiamentos: Não há.

Considerações éticas: Este trabalho não necessitou ser submetido previamente a conselho de ética.

Agradecimento/Contribuições adicionais: Os autores agradecem aos participantes das oficinas pela grande contribuição na construção coletiva deste estudo.

Histórico do artigo: Submetido: 09.mar.2016 | Aceito: 18.jul.2016 | Publicado: 30.set.2016.

Apresentação anterior: Não há.

Licença CC BY-NC atribuição não comercial. Com essa licença é permitido acessar, baixar (download), copiar, imprimir, compartilhar, reutilizar e distribuir os artigos, desde que para uso não comercial e com a citação da fonte, conferindo os devidos créditos de autoria e menção à Reciis. Nesses casos, nenhuma permissão é necessária por parte dos autores ou dos editores. 


\section{Introdução}

Em virtude das transições demográfica e epidemiológica que vêm ocorrendo em âmbito mundial, tem-se presenciado um envelhecimento da população aliado a uma queda relativa dos agravos agudos e a um aumento crescente das condições crônicas de saúde ${ }^{1}$. No Brasil, estas mudanças vêm ocorrendo de forma significativa a partir da segunda metade do século XX, e o país vivencia atualmente uma transição epidemiológica com tripla carga de doenças em que, segundo Mendes $^{2-3}$, persistem doenças infecciosas, desnutrição e problemas de saúde reprodutiva concomitantemente com o incremento das doenças crônicas e de seus fatores de risco, além do forte crescimento das causas externas de morbimortalidade.

As Redes de Atenção à Saúde (RAS) surgem, no âmbito do Sistema Único de Saúde (SUS), como uma alternativa ao modelo assistencial caracterizado pela sua fragmentação, que resulta em um conjunto de pontos de atenção à saúde isolados e sem comunicação entre si e, portanto, incapazes de prestar uma atenção contínua e integral à população. As RAS, constituídas pela população, a estrutura operacional e o modelo de atenção à saúde 3 são o conjunto de ações e serviços de saúde articulados em níveis de complexidade crescente, com a finalidade de garantir a integralidade da assistência à saúde4 .

As RAS estão compreendidas no âmbito de uma Região de Saúde, ou de várias delas, em consonância com diretrizes pactuadas nas Comissões Intergestores. De acordo com o Decreto $n^{0} 7508$, de 2011, a Região de Saúde é "o espaço geográfico contínuo constituído por agrupamentos de municípios limítrofes, delimitado a partir de identidades culturais, econômicas e sociais e de redes de comunicação e infraestrutura de transportes compartilhados" . A regionalização, de acordo Viana e Lima", "é um processo político que envolve mudanças na distribuição de poder e o estabelecimento de um sistema de inter-relações entre diferentes atores sociais" inseridos em espaços geográficos delimitados e que, por conseguinte, demanda "a formulação e implementação de estratégias e instrumentos de planejamento, integração, gestão, regulação e financiamento de uma rede de ações e serviços no território”. Desse modo, as RAS se apresentam como uma proposta integradora com capacidade para dar respostas positivas à nova situação sanitária. Conforme Mendes $^{3}$, existem experiências internacionais de sucesso nesse sentido com melhora de resultados sanitários e econômicos, as quais poderiam ser adotadas e adaptadas à realidade do sistema público de saúde brasileiro

Assim, entre as diretrizes orientadoras do processo de implementação das RAS estão o desenvolvimento de sistemas de apoio que têm como estratégias: a promoção de comunicação de todos os pontos de atenção com a implementação de Registro Eletrônico em Saúde (RES); o avanço no desenvolvimento da gestão da tecnologia de informação e comunicação em saúde; e a utilização de sistemas de informação em saúde (SIS) como ferramentas importantes para construção do diagnóstico da situação de saúde, a fim de produzir intervenções baseadas nas necessidades das populações ${ }^{4}$.

No Brasil, as primeiras experiências com as RAS ocorreram no início do século XXI e se disseminaram pelo país, em geral sob a coordenação das secretarias de Saúde estaduais e sob a forma de redes temáticas. As diretrizes para a sua organização, no âmbito do SUS, foram publicadas em 2010, pela Portaria GM/MS n. ${ }^{\circ} 4279$, e definidas como uma "estratégia para superar a fragmentação da atenção e da gestão nas Regiões de Saúde e aperfeiçoar o funcionamento político-institucional do SUS, com vistas a assegurar ao usuário o conjunto de ações e serviços que necessita com efetividade e eficiência"4.

A avaliação do desempenho das RAS aparece nesse contexto como uma questão inerente à gestão, de forma a possibilitar a verificação da coerência entre mudanças no modelo de atenção e as necessidades da população. Para avaliação de desempenho de sistemas de saúde, Viacava e colaboradores apresentaram, como resultado do Projeto de Avaliação de Desempenho do Sistema de Saúde (Proadess), uma proposta de Matriz das dimensões de avaliação, na qual os indicadores estão estruturados em quatro dimensões: determinantes da saúde, condições de saúde da população, sistema de saúde e desempenho dos serviços de saúde. 
Embora o desempenho dos serviços de saúde esteja condicionado à estrutura do sistema, que por sua vez deve ser orientado pelos determinantes e condições de saúde, o objeto de análise deste artigo está restrito à dimensão do desempenho dos serviços de saúde no contexto das RAS. Esta escolha justifica-se pelo fato de ser esta dimensão a que influencia mais diretamente a atenção à saúde prestada, preservando assim o enfoque nos resultados da situação de saúde da população.

A literatura a respeito de estudos sobre o tema de avaliação de desempenho das RAS no Brasil é escassa, com uma lacuna nas normativas acerca da definição de mecanismos de monitoramento e avaliação de desempenho. Com o propósito de identificar uma ferramenta que permita a avaliação do desempenho das RAS, os autores partiram do marco conceitual das RAS proposto por Mendes ${ }^{2}$ associado à aplicação de indicadores de desempenho propostos pelo Proadess.

Todavia, a avaliação de desempenho das RAS deve ser apoiada por informações de qualidade provindas de SIS confiáveis, conforme destacado por Mendes². O grande volume de dados captados nas RAS possibilita a instituição de instrumentos para essa avaliação, implicando na apropriação sistemática e dinâmica dos dados produzidos no processo de atenção à saúde, transformando-os de simples registros burocráticos em "informação gerencial".

A experiência brasileira em SIS remonta aos anos de $1960^{\circ}$. Ao longo das últimas três décadas, diversos SIS contendo dados epidemiológicos, demográficos, assistenciais e administrativos de base nacional foram estruturados no âmbito do SUS e amplamente disponibilizados para serem usados na construção de indicadores para avaliação de desempenho das RAS ${ }^{3}$.

No entanto, conforme apontado por Panitz ${ }^{10}$, o modo como esses SIS foram instituídos reflete o próprio sistema de atenção à saúde fragmentado, apresentando, por vezes, o que Mendes 3 tem denominado de "[...] problemas de excessividade injustificada e de baixa qualidade [...]”. Ademais, a ausência de padronização técnica e semântica dos instrumentos de coleta, incluindo campos comuns de identificação, bem como a concepção de sistemas que priorizam os objetivos administrativo-financeiros, em detrimento da avaliação do cuidado em saúde, limitam as potencialidades dos diferentes SIS. A ausência de integração pode ter impacto na qualidade das informações geradas e, por consequência, nos indicadores de desempenho construídos com base nesses SIS.

É necessário, portanto, verificar se as informações disponíveis atendem às necessidades para a construção de indicadores de avaliação de desempenho das RAS. Nesse contexto, o presente artigo busca responder à seguinte questão norteadora: como avaliar o desempenho das RAS a partir de indicadores provenientes de SIS de base nacional? Buscou-se, então, elaborar uma proposta de avaliação de desempenho mediante a identificação de um agravo de saúde que perpasse, na sua linha de cuidado, os níveis de complexidade da atenção. Tal agravo funcionaria, numa perspectiva sistêmica, como um evento sentinela que permitiria a avaliação da rede incluindo instâncias de apoio, tais como os laboratórios e os próprios SIS, por meio de indicadores que deverão atentar à necessidade de funcionamento integrado dos elementos das redes com o objetivo de agregar valor à população em termos de melhores resultados de saúde ${ }^{11}$, motivo pelo qual se entende o presente estudo como relevante para a área social e política no modelo de regionalização do SUS.

\section{Estratégia metodológica}

Com o intuito de selecionar o agravo e os respectivos indicadores de desempenho a serem abordados no estudo descritivo de processo metodológico de natureza operacional foram realizadas três oficinas de trabalho. A opção pelas oficinas como estratégias metodológicas de pesquisa decorreu do entendimento de que esses espaços têm "potencial crítico de negociação de sentidos, permitindo visibilidade de argumentos, posições, mas também deslocamentos, construção e contraste de versões" ${ }^{12}$. 
A oficina I teve o seu planejamento estruturado em formato de roteiro, que continha os objetivos e resultados esperados, além da descrição das atividades, a dinâmica de trabalho, agenda, matrizes de indicação de agravos de saúde e de priorização de agravos de saúde e documentação de apoio. O objetivo foi selecionar agravos de saúde que atendessem critérios definidos previamente para análise de desempenho das RAS no SUS por meio da utilização de uma metodologia participativa geradora de consenso, a Técnica de Grupo Nominal (TGN). A TGN foi desenvolvida na década de 1960 nos Estados Unidos da América para pesquisas sociais na área de psicologia e permite selecionar, fazer julgamentos e desenvolver a criatividade de sugestões para a resolução de um problema. Essa técnica vem sendo aplicada em diversas áreas como saúde, educação, serviços sociais, indústria e governo ${ }^{13-14}$.

A TGN está estruturada para a geração de ideias coletivas por meio do incentivo à participação equilibrada, minimizando as diferenças e permitindo a interação dos diversos saberes envolvidos no assunto. É utilizada nos casos em que o tempo é um limitador para escolha de opções, tendo o objetivo de gerar consenso para a tomada de decisões ${ }^{15}$. É um método que reúne especialistas escolhidos sobre o tema, também conhecido por Painel de Especialistas, com cerca de 9 a 12 participantes, usualmente ${ }^{16}$. O termo nominal refere-se a processos que reúnem os indivíduos, mas prescinde do debate entre eles ${ }^{17-18}$.

A TGN possibilita abordagens qualitativas não consideradas em métodos quantitativos, por considerar opiniões de especialistas para resolução de problemas. Expressa as preferências do grupo, com base em uma resposta construída por meio do anonimato, a respeito do que deve ser priorizado; a aplicação em etapas permite aos participantes a mudança de opiniões; além disso, a TGN propicia o retorno dos resultados de forma imediata e direta aos participantes por medidas estatísticas utilizadas na priorização $0^{14}$.

Originalmente consiste em cinco etapas, que se iniciam com as ideias geradoras, passam pela gravação de ideias, discussão, votação, e finalizam com a soma das votações ${ }^{14}$. A TGN, todavia, pode ser adaptada em relação às etapas ${ }^{16}$. Dessa forma, para o presente estudo, a técnica foi aplicada seguindo sete passos: (1) definição de papéis (relator, coordenador e cronometrador), (2) objetivos, (3) chuva de ideias individuais, (4) intercâmbio e registro de ideias, (5) discussão em grupo, (6) priorização individual pontuada com base nos critérios definidos, e (7) conclusão. O detalhamento dos conceitos e as atividades de cada passo foram esclarecidos aos participantes conforme descrição da dinâmica constante no roteiro da oficina.

Neste estudo, algumas reuniões de grupo nominal incorporaram revisão da literatura como material de apoio para o tópico em discussão ${ }^{16}$. O material de apoio foi estruturado pela equipe de pesquisa e compartilhado com os convidados em meio eletrônico, previamente à realização da oficina, com a utilização da ferramenta de compartilhamento online 'Dropbox' e continha indicação de leituras e material consolidado sobre as linhas de cuidado, diretrizes e políticas nacionais.

A Oficina I teve oito participantes, incluindo convidados de acordo com critérios de envolvimento profissional ou acadêmico no âmbito das RAS e a equipe envolvida diretamente na pesquisa, com atuação nas seguintes categorias: médico com experiência na atenção primária; enfermeiro da rede nível ambulatorial; técnica da área de vigilância em saúde; aluno de pós-graduação em Saúde Coletiva - nível mestrado profissional; docentes das áreas de planejamento, gestão e avaliação, epidemiologia e promoção da saúde.

A dinâmica se desenvolveu por meio da atuação de um facilitador que conduziu o debate junto aos especialistas, em uma reunião estruturada na qual os participantes relataram as suas opiniões e propostas, além de realizar a discussão e priorização junto ao grupo ${ }^{15}$. Seu objetivo foi determinar até que ponto os especialistas concordavam sobre determinado assunto, procurando superar desvantagens encontradas em reuniões de grupos dominadas por indivíduos ou com a existência de interesses conflitantes com dificuldade de consenso ${ }^{16}$.

O resultado esperado era a seleção de dois agravos de saúde que tivessem a atenção básica (AB) como porta de entrada principal ao sistema, sendo priorizado para objeto deste estudo o agravo que teve maior 
pontuação. Os critérios para seleção de agravos, definidos previamente pela equipe de pesquisa do projeto, foram os seguintes: (1) utilização da $\mathrm{AB}$ como porta de entrada principal no sistema; (2) magnitude; (3) possuir linhas de cuidado ou diretrizes preestabelecidas pelo Ministério da Saúde (MS); (4) perpassar todos os níveis de atenção, desde a prevenção até tratamento e reabilitação; e (5) existência de SIS que permitissem o seu monitoramento. Um critério adicional, incluído por sugestão unânime dos participantes, foi que o agravo constituísse uma condição sensível à $\mathrm{AB}$.

Com o propósito de atender à atividade de identificação dos indicadores de desempenho para o agravo de saúde priorizado na Oficina I foram realizadas as Oficinas II e III. O resultado esperado era um elenco de indicadores de desempenho selecionados para o agravo, de acordo com os componentes da estrutura operacional das RAS definida por Mendes (Figura 1) ${ }^{3}$ e classificados segundo as seguintes subdimensões de desempenho propostas no modelo de avaliação de Viacava e colaboradores7:

(1) efetividade; (2) acesso; (3) eficiência; (4) respeito ao direito das pessoas; (5) aceitabilidade; (6) continuidade; (7) adequação; (8) segurança. Para este estudo, em uma proposta de adequação à avaliação das RAS, foram selecionadas as de números 1, 2, 6 e 7, por ser possível mensurá-las em sistema de indicadores com base em SIS nacionais.

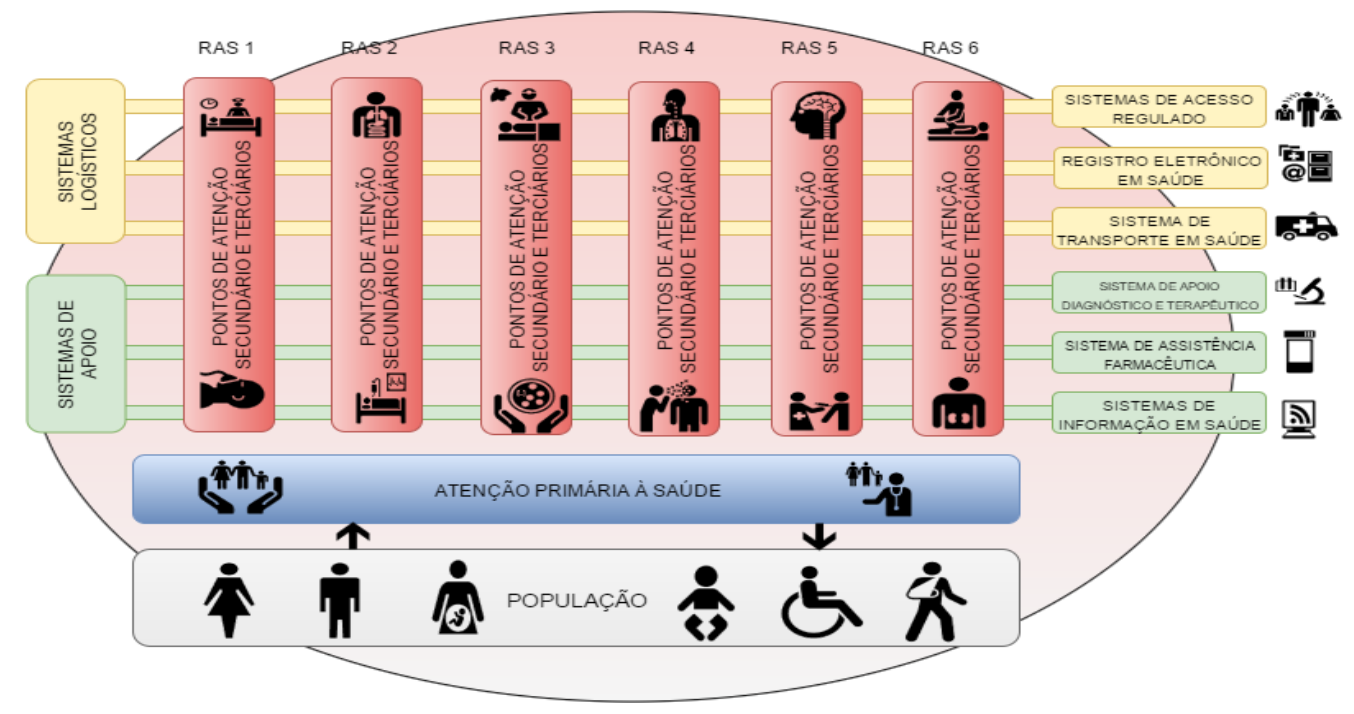

Figura 1: Estrutura operacional das RAS Fonte: Adaptado de Mendes (2011).

As dimensões selecionadas são definidas como:

- Efetividade: grau com que a assistência, os serviços e as ações atingem os resultados esperados;

- Acesso: capacidade do sistema de saúde para prover o cuidado e o serviço necessários, no momento certo e no lugar adequado;

- Continuidade: capacidade do sistema de saúde prestar serviços de forma ininterrupta e coordenada entre diferentes níveis de atenção;

- Adequação: grau com que os cuidados prestados às pessoas estão baseados no conhecimento técnico-científico existente ${ }^{19}$.

A proposta de seleção dos indicadores foi detalhada no roteiro por meio de uma matriz estruturada adotando conceitos da Ficha de qualificação do indicador, recomendados pela Rede Interagencial de Informações para a 
Saúde (RIPSA), sendo esta “o principal instrumento de orientação técnica [...] que esclarece os conceitos e critérios adotados [...] para os indicadores" ${ }^{20}$.

Os mesmos componentes da Oficina I foram convidados para participar da Oficina II e fizeram a indicação conjunta de indicadores de desempenho do agravo priorizado, preenchendo a matriz com o nome do indicador, a estrutura operacional da RAS a que se refere, a subdimensão do desempenho e o método de cálculo. As informações adicionais da matriz foram preenchidas pela equipe do projeto. As metas de desempenho foram discutidas conforme os pontos de corte adequados a cada indicador.

A Oficina III, para validação qualitativa e seleção final dos indicadores, contou com a participação da equipe do projeto de pesquisa e de um pesquisador com experiência na avaliação de desempenho do sistema de saúde brasileiro e qualidade de vida.

Para validação qualitativa final foram consideradas as propriedades essenciais de um indicador, ou seja, aquelas que qualquer indicador de programa deve apresentar e que sempre devem ser consideradas como critérios de escolha: validade, confiabilidade e simplicidade. As propriedades complementares de um indicador, tais como a sensibilidade, desagregabilidade, economicidade, estabilidade, mensurabilidade e auditabilidade foram consideradas de forma secundária ${ }^{21}$. Além das propriedades levadas em conta, a análise para validação qualitativa também considerou a necessidade de pertencerem a uma das subdimensões de desempenho definidas para o estudo ${ }^{7}$, a um dos componentes da estrutura operacional da RAS ${ }^{3}$, assim como possuírem recomendações pelas Diretrizes de controle do câncer de colo de útero ${ }^{22}$ e fontes de informações disponíveis, regulares e confiáveis para o seu cálculo em base nacional.

Após a validação qualitativa final, realizou-se o detalhamento dos indicadores selecionados por meio da construção das fichas de qualificação. Esse detalhamento foi realizado seguindo os padrões adotados pela RIPSA ${ }^{20}$.

\section{Resultados}

Na Oficina I fez-se a identificação de agravo de saúde pública que permitisse a análise de desempenho das RAS em todos os seus níveis de atenção. Após a definição dos papéis de relator, coordenador e cronometrador (passo 1) e dos objetivos da TGN (passo 2), iniciou-se a chuva de ideias individual (passo 3). Cada participante, de forma individual, indicou quatro agravos de saúde que tivessem a $\mathrm{AB}$ como porta de entrada principal no sistema. Nessa primeira rodada, os participantes anotaram individualmente suas indicações na "Matriz de indicação de agravos de saúde", marcando os critérios atendidos por cada uma delas.

Em seguida, fez-se o intercâmbio e registro de ideias (passo 4), com a anotação das indicações individuais no quadro pelo relator, agrupando-se as indicações repetidas ou similares. Cada participante argumentou acerca das suas indicações, porém sem perguntas, discussão e debate. Todas as ideias foram aceitas, sem exceção, chegando-se ao resultado de seis agravos com a AB como porta de entrada principal no sistema: sífilis congênita, diabetes mellitus, hipertensão arterial sistêmica, câncer de mama, câncer do colo do útero e anemia falciforme.

Na discussão em grupo (passo 5), os participantes puderam realizar perguntas e tornar claras as argumentações realizadas para as indicações, sendo possível nessa etapa uma análise em grupo das vantagens e desvantagens de cada indicação e o debate que permitiu a reorganização.

Como o resultado esperado era a priorização de dois agravos de saúde, partiu-se para a priorização individual (passo 6). Nessa segunda rodada, cada participante, de forma individual, classificava e ordenava os agravos de acordo com a importância que lhes atribuía, priorizando, entre as indicações realizadas pelo grupo, dois agravos que tivessem a $\mathrm{AB}$ como porta de entrada principal no sistema.

Os participantes utilizaram a "Matriz de priorização de agravos de saúde" para listar as principais vantagens e desvantagens que os levaram à escolha e atribuindo pontuações entre 1 e 2 para os agravos selecionados, em ordem crescente de priorização. O relator anotou no quadro as pontuações individuais 
atribuídas pelos participantes para cada agravo, sendo considerado coletivamente com máxima prioridade o agravo que recebesse a pontuação mais alta.

Na conclusão (passo 7), o relator calculou a pontuação final de cada agravo e apresentou a classificação final tabulada. O câncer do colo do útero obteve a maior pontuação, com 12 pontos de 14 possíveis, seguido pela hipertensão arterial sistêmica com quatro pontos. O quadro final de agravos indicados e agravo priorizado pode ser visualizado no Quadro 1.

Quadro 1 - Matriz de consolidação de indicação e priorização de agravos de saúde

\begin{tabular}{|c|c|c|}
\hline \multirow{2}{*}{ Agravos indicados } & \multicolumn{2}{|c|}{ Agravos priorizados } \\
\hline & Priorização & Pontuação total \\
\hline \multicolumn{3}{|c|}{ Atenção básica } \\
\hline Sífilis Congênita & $2+1$ & 3 \\
\hline Diabetes Mellitus & 1 & 1 \\
\hline Hipertensão Arterial Sistêmica & $1+1+1+1$ & 4 \\
\hline Câncer de Mama & 1 & 1 \\
\hline Câncer do Colo do Útero & $2+2+2+2+2+2$ & 12 \\
\hline Anemia Falciforme & 0 & 0 \\
\hline
\end{tabular}

Fonte: Elaborado pelos autores.

A seleção dos principais indicadores para monitoramento e avaliação do desempenho das RAS foi iniciada por meio da Oficina II, a partir de uma revisão dos agravos priorizados na Oficina I e da apresentação da Matriz de indicação de indicadores de desempenho. Foram sugeridos 18 indicadores para a prevenção, diagnóstico e manejo do câncer do colo do útero conforme Quadro 2.

Quadro 2 - Indicadores sugeridos na Oficina II

\begin{tabular}{|l|}
\hline Nome do indicador \\
\hline Razão entre exames citopatológicos de colo do útero na faixa etária de 25 a 64 anos em relação à população-alvo \\
\hline Mulheres cobertas pelo programa \\
\hline Coletas anuais na mesma mulher \\
\hline Coletas bianuais na mesma mulher \\
\hline Recepção de exames citopatológicos de colo do útero \\
\hline Lâminas rejeitadas \\
\hline Amostras insatisfatórias \\
\hline Exames realizados \\
\hline Disponibilização do resultado do exame \\
\hline Resultados entregues em até 30 dias \\
\hline Exames normais \\
\hline Exames alterados \\
\hline Procedimento realizado \\
\hline Exames indicativos de câncer \\
\hline Exame citopatológico de colo do útero positivo encaminhado para biópsia com menos de 30 dias \\
\hline Biópsia ou Tratamento in sito \\
\hline Encaminhamento para atenção hospitalar \\
\hline Realização de quimioterapia ou radioterapia \\
\hline
\end{tabular}


Após o término da carga horária da Oficina II, pactuou-se com os participantes que a equipe do projeto realizaria, até a data da Oficina III, a finalização da sugestão dos indicadores para o agravo do câncer do colo do útero. A finalização seguiu as diretrizes da Política Nacional de Controle e Prevenção do Câncer do Ministério da Saúde (MS) e as ações acompanhadas e recomendadas pelo Instituto Nacional do Câncer (Inca).

Por meio da revisão documental, alguns indicadores foram renomeados, outros substituídos ou acrescentados para adequação às recomendações das diretrizes do controle do câncer do colo do útero do Inca ${ }^{22}$. Os indicadores renomeados foram "Mulheres cobertas pelo programa", "Lâminas rejeitadas", "Amostras insatisfatórias", "Resultados entregues em até 30 dias" e "Exames alterados", definidos respectivamente como "Cobertura de exames citopatológicos do colo do útero em mulheres da populaçãoalvo de 25 a 64 anos", "Percentual de exames rejeitados", "Percentual de amostras insatisfatórias", "Proporção de exames citopatológicos do colo do útero liberados em até 30 dias" e "Índice de positividade".

O indicador "Procedimento realizado" foi substituído por "Proporção de tratamentos iniciados em até 60 dias após a confirmação do diagnóstico de neoplasia”, uma vez que não estava claro a quais procedimentos tinham se referido na sugestão inicial. Ademais, a Lei $\mathrm{n}^{0} 12.732$ de $2012^{23}$ definiu o prazo de 60 dias para início do tratamento após a confirmação diagnóstica de neoplasia, considerando para fins de tratamento efetivamente iniciado a realização de terapia cirúrgica, quimioterapia ou radioterapia.

O indicador "Exames indicativos de câncer" foi desmembrado em dois: "Razão entre lesão de alto grau e carcinoma epidermoide invasivo em exames citopatológicos de colo do útero" e "Razão entre exames histopatológicos de colo do útero com diagnóstico NIC III e carcinoma invasor". A justificativa para essa substituição reside no fato de serem um detalhamento dos resultados diagnósticos indicativos de neoplasia por meio dos exames citopatológicos e histopatológicos, acompanhados no Painel de indicadores do câncer do colo do útero do Inca' ${ }^{22}$.

O indicador de "Proporção de seguimento informado para mulheres com lesão intraepitelial de alto grau do colo do útero" foi acrescentado por tratar-se do único indicador de diagnóstico e tratamento recomendado pelo Inca por meio da Ficha técnica de indicadores das ações de controle do câncer do colo do útero, sendo "fundamental para avaliação das ações do programa de controle deste câncer"24.

O "Percentual sem citologia anterior", "Percentual com citologia anterior" e "Percentual sem informação se houve citologia anterior" foram acrescentados nas sugestões por serem indicadores monitorados pelo Inca, por meio do Painel de indicadores do câncer do colo do útero. Esses indicadores podem sinalizar a capacidade da rede de realizar exames pela primeira vez, verificar a repetição de exames fora da periodicidade e a necessidade de capacitações quanto ao preenchimento dos formulários e a qualidade da informação, respectivamente ${ }^{22}$.

Os indicadores "Tempo de investigação diagnóstica", "Percentual de exames de rastreamento na faixa etária-alvo" e "Mulheres da população-alvo que realizaram exames de rastreamento" foram acrescentados por possuírem informações com base em relatórios gerenciais disponíveis no Sistema de Informação do Câncer (Siscan). Esses relatórios são atualizados de acordo com as informações registradas no sistema online.

A sugestão final de indicadores foi submetida à validação qualitativa na Oficina III. Foi apresentada como sugestão uma relação com um total de 26 indicadores de desempenho referentes ao agravo do câncer do colo do útero, conforme Quadro 3.

Partiu-se então para a etapa de aplicação dos critérios para a validação qualitativa, realizando uma análise pontual de cada indicador selecionado. Nessa etapa, alguns indicadores foram desconsiderados no presente estudo por não atenderem alguns dos critérios de validação definidos, conforme detalhamento a seguir:

1. Percentual sem citologia anterior, Tempo de investigação diagnóstica, Percentual de exames de rastreamento na faixa etária-alvo, Mulheres da população-alvo que realizaram exames de rastreamento e não possuíam as propriedades essenciais de um indicador; 
2. Exames realizados, Exames normais, Exame citopatológico de colo do útero positivo encaminhado para biópsia em menos de 30 dias, Biópsia ou Tratamento in sito, Encaminhamentos para atenção hospitalar, Realização de quimioterapia ou radioterapia, Percentual com citologia anterior, Percentual sem informação se houve citologia anterior não estavam adequados às recomendações atualizadas das Diretrizes de controle do câncer do colo do útero do Inca ${ }^{22}$.

3. Coletas anuais na mesma mulher, Coletas bianuais na mesma mulher, Recepção de exames citopatológicos de colo do útero, Disponibilização do resultado do exame, Proporção de segmento informado para mulheres com lesão intraepitelial de alto grau do colo do útero não possuíam informações disponíveis, regulares e confiáveis para o seu cálculo em base nacional:

Quadro 3 - Indicadores selecionados para validação qualitativa

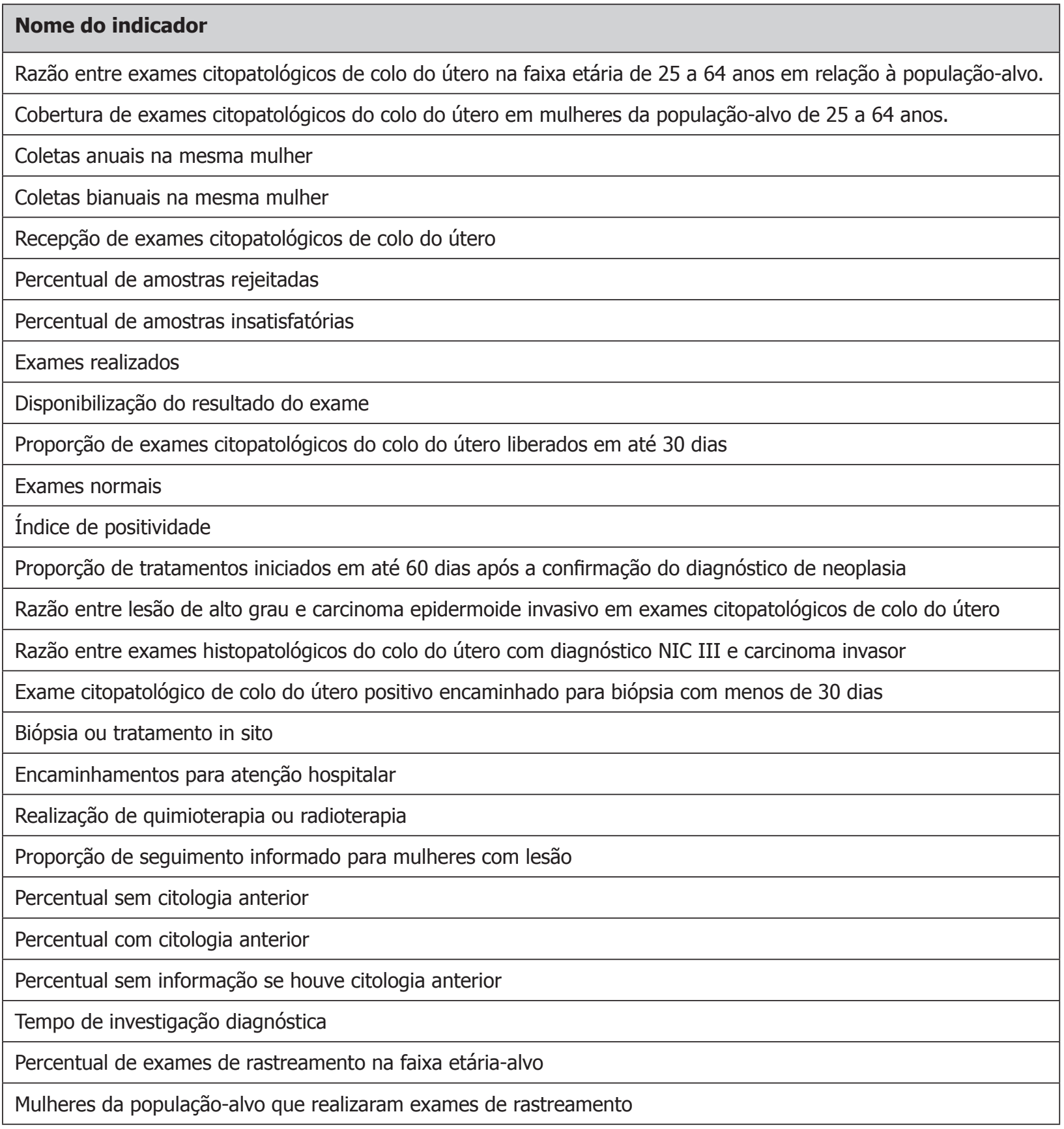

Fonte: Elaborado pelos autores. 
Tendo em vista a necessidade de análise da linha de cuidado do agravo em sua completude, desde o diagnóstico até o desfecho final, incluiu-se o indicador de Taxa de mortalidade por câncer do colo do útero que, embora não tivesse sido sugerido nas oficinas, foi considerado um indicador de desfecho importante a ser avaliado, inclusive pelo fato de que o óbito é o único desfecho possível via SIS para o câncer do colo do útero, uma vez que pacientes com diagnóstico confirmado permanecem em acompanhamento depois do tratamento. Ademais, o indicador em questão é um dos indicadores de resultado e impacto recomendados pelo Inca ${ }^{22}$.

Chegou-se a uma composição final de 10 indicadores validados para o presente estudo, distribuídos entre as subdimensões de desempenho e os componentes da estrutura operacional da RAS definidos: Centro Comunicador, Sistemas de Apoio e Pontos de Atenção Secundários e Terciários (Quadro 4).

Quadro 4 - Indicadores de desempenho do câncer do colo do útero validados

\begin{tabular}{|c|c|c|c|c|}
\hline $\begin{array}{l}\text { Estrutura } \\
\text { Operacional da } \\
\text { RAS }\end{array}$ & $\begin{array}{l}\text { Subdimensão } \\
\text { do } \\
\text { desempenho }\end{array}$ & $\begin{array}{l}\text { Nome do } \\
\text { indicador }\end{array}$ & Método de Cálculo & $\begin{array}{l}\text { Fonte de } \\
\text { Informação }^{1}\end{array}$ \\
\hline $\begin{array}{l}\text { Centro } \\
\text { Comunicador }\end{array}$ & Acesso & $\begin{array}{l}\text { Razão de exames } \\
\text { citopatológicos } \\
\text { do colo do útero } \\
\text { na faixa etária } \\
\text { de } 25 \text { a } 64 \text { anos } \\
\text { e a população } \\
\text { feminina na } \\
\text { mesma faixa } \\
\text { etária }\end{array}$ & $\begin{array}{l}\text { N. }{ }^{\circ} \text { total de exames } \\
\text { citopatológicos do colo do útero } \\
\text { apresentados, na faixa etária de } \\
25 \text { a } 64 \text { anos, dos residentes em } \\
\text { determinado local em período/ } \\
\text { (População feminina na faixa } \\
\text { etária de } 25 \text { a } 64 \text { anos, no mesmo } \\
\text { local e período/3) }\end{array}$ & SIA; IBGE. \\
\hline $\begin{array}{l}\text { Centro } \\
\text { Comunicador }\end{array}$ & $\begin{array}{l}\text { Acesso/ } \\
\text { Adequação }\end{array}$ & $\begin{array}{l}\text { Cobertura } \\
\text { de exames } \\
\text { citopatológicos } \\
\text { do colo do útero } \\
\text { em mulheres da } \\
\text { população-alvo de } \\
25 \text { a } 64 \text { anos }\end{array}$ & $\begin{array}{l}\text { No total de mulheres, na faixa } \\
\text { etária de } 25 \text { a } 64 \text { anos, que } \\
\text { realizaram exame citopatológico } \\
\text { do colo do útero nos últimos três } \\
\text { anos, residentes em determinado } \\
\text { local em período/População } \\
\text { feminina residente na faixa etária } \\
\text { de } 25 \text { a } 64 \text { anos, no mesmo local } \\
\text { e período }\end{array}$ & Siscan, IBGE \\
\hline $\begin{array}{l}\text { Centro } \\
\text { Comunicador }\end{array}$ & $\begin{array}{l}\text { Adequação/ } \\
\text { Continuidade }\end{array}$ & $\begin{array}{l}\text { Percentual } \\
\text { de amostras } \\
\text { rejeitadas }\end{array}$ & $\begin{array}{l}\mathrm{N} .^{\circ} \text { total de amostras rejeitadas } \\
\text { do exame citopatológico do } \\
\text { colo do útero liberadas, em } \\
\text { determinado local e período/N. }{ }^{\circ} \\
\text { total de exames citopatológicos do } \\
\text { colo do útero liberados no mesmo } \\
\text { local período } * 100\end{array}$ & Siscan \\
\hline $\begin{array}{l}\text { Centro } \\
\text { Comunicador }\end{array}$ & $\begin{array}{l}\text { Adequação/ } \\
\text { Continuidade }\end{array}$ & $\begin{array}{l}\text { Percentual } \\
\text { de amostras } \\
\text { insatisfatórias }\end{array}$ & $\begin{array}{l}\mathrm{N}{ }^{\circ}{ }^{\circ} \text { total de amostras } \\
\text { insatisfatórias do exame } \\
\text { citopatológico do colo do útero } \\
\text { liberadas, em determinado local } \\
\text { e período/ } \mathrm{N}^{\circ} \text { total de exames } \\
\text { citopatológicos do colo do útero } \\
\text { liberados no mesmo local e } \\
\text { período } * 100\end{array}$ & Siscan \\
\hline
\end{tabular}

Fonte: Elaborado pelos autores.

1 - Fonte de Informação: Sistema de Informação Ambulatorial do Sistema Único de Saúde (SIA); Censo Populacional do Instituto Brasileiro de Geografia e Estatística (IBGE); Sistema de Informação do Câncer do Sistema Único de Saúde (Siscan); Sistema de Informação de Mortalidade (SIM). 
Quadro 4 - Indicadores de desempenho do câncer do colo do útero validados

\begin{tabular}{|c|c|c|c|c|}
\hline $\begin{array}{l}\text { Estrutura } \\
\text { Operacional da } \\
\text { RAS }\end{array}$ & $\begin{array}{l}\text { Subdimensão } \\
\text { do } \\
\text { desempenho }\end{array}$ & $\begin{array}{l}\text { Nome do } \\
\text { indicador }\end{array}$ & Método de Cálculo & $\begin{array}{l}\text { Fonte de } \\
\text { Informação }^{1}\end{array}$ \\
\hline Sistemas de apoio & $\begin{array}{l}\text { Adequação/ } \\
\text { Continuidade/ } \\
\text { Efetividade }\end{array}$ & $\begin{array}{l}\text { Proporção } \\
\text { de exames } \\
\text { citopatológicos } \\
\text { do colo do útero } \\
\text { liberados em até } \\
30 \text { dias }\end{array}$ & $\begin{array}{l}\text { No total de exames citopatológicos } \\
\text { do colo do útero satisfatórios } \\
\text { com resultados liberados em } \\
\text { até } 30 \text { dias após a coleta, em } \\
\text { determinado local e período/N.o } \\
\text { total de exames citopatológicos } \\
\text { do colo do útero satisfatórios } \\
\text { liberados, no mesmo local e } \\
\text { período } * 100\end{array}$ & Siscan \\
\hline Sistemas de apoio & Adequação & $\begin{array}{l}\text { Índice de } \\
\text { positividade }\end{array}$ & $\begin{array}{l}\text { No total de exames citopatológicos } \\
\text { do colo do útero liberados } \\
\text { com resultados alterados, em } \\
\text { determinado local e período/No } \\
\text { total de exames citopatológicos } \\
\text { do colo do útero satisfatórios } \\
\text { liberados, no mesmo local e } \\
\text { período } * 100\end{array}$ & Siscan \\
\hline Sistemas de apoio & $\begin{array}{l}\text { Acesso/ } \\
\text { Adequação/ } \\
\text { Efetividade }\end{array}$ & $\begin{array}{l}\text { Razão entre } \\
\text { lesão de alto } \\
\text { grau e carcinoma } \\
\text { epidermoide } \\
\text { invasivo } \\
\text { em exames } \\
\text { citopatológicos de } \\
\text { colo do útero. }\end{array}$ & $\begin{array}{l}\text { N. }{ }^{\circ} \text { total de exames } \\
\text { citopatológicos do colo do útero } \\
\text { liberados com resultado de lesão } \\
\text { de alto grau, em determinado } \\
\text { local e período/ N. }{ }^{\circ} \text { total de } \\
\text { exames citopatológicos do colo do } \\
\text { útero liberados com resultado de } \\
\text { carcinoma epidermoide invasivo, } \\
\text { no mesmo local e período }\end{array}$ & Siscan \\
\hline Sistemas de apoio & $\begin{array}{l}\text { Acesso/ } \\
\text { Adequação/ } \\
\text { Efetividade }\end{array}$ & $\begin{array}{l}\text { Razão entre } \\
\text { exames } \\
\text { histopatológicos } \\
\text { do colo do útero } \\
\text { com diagnóstico } \\
\text { NIC III e } \\
\text { carcinoma invasor }\end{array}$ & $\begin{array}{l}\text { N. }{ }^{\circ} \text { total de exames } \\
\text { histopatológicos do colo do } \\
\text { útero liberados com resultado de } \\
\text { NIC III, em determinado local } \\
\text { e período/N. }{ }^{\circ} \text { total de exames } \\
\text { histopatológicos do colo do } \\
\text { útero liberados com resultado de } \\
\text { carcinoma invasor, no mesmo local } \\
\text { e período }\end{array}$ & Siscan \\
\hline $\begin{array}{l}\text { Pontos de } \\
\text { Atenção } \\
\text { Secundários e } \\
\text { Terciários }\end{array}$ & $\begin{array}{l}\text { Acesso/ } \\
\text { Adequação/ } \\
\text { Continuidade/ } \\
\text { Efetividade }\end{array}$ & $\begin{array}{l}\text { Proporção de } \\
\text { tratamentos } \\
\text { iniciados em até } \\
60 \text { dias após a } \\
\text { confirmação do } \\
\text { diagnóstico de } \\
\text { neoplasia }\end{array}$ & $\begin{array}{l}\text { N. }{ }^{\circ} \text { total de tratamentos iniciados } \\
\text { em até } 60 \text { dias após a confirmação } \\
\text { do diagnóstico de neoplasia do } \\
\text { colo do útero, em determinado } \\
\text { local e período/N. }{ }^{\circ} \text { total de } \\
\text { diagnósticos confirmados de } \\
\text { neoplasia do colo do útero, no } \\
\text { mesmo local e período } * 100\end{array}$ & Siscan \\
\hline $\begin{array}{l}\text { Pontos de } \\
\text { Atenção } \\
\text { Secundários e } \\
\text { Terciários }\end{array}$ & $\begin{array}{l}\text { Acesso/ } \\
\text { Adequação/ } \\
\text { Continuidade/ } \\
\text { Efetividade }\end{array}$ & $\begin{array}{l}\text { Taxa de } \\
\text { mortalidade por } \\
\text { câncer do colo do } \\
\text { útero }\end{array}$ & $\begin{array}{l}\text { No total de óbitos por câncer do } \\
\text { colo do útero, em determinado } \\
\text { local e ano / População feminina, } \\
\text { no mesmo local e ano X } 100.000\end{array}$ & SIM, IBGE \\
\hline
\end{tabular}

Fonte: Elaborado pelos autores.

1 - Fonte de Informação: Sistema de Informação Ambulatorial do Sistema Único de Saúde (SIA); Censo Populacional do Instituto Brasileiro de Geografia e Estatística (IBGE); Sistema de Informação do Câncer do Sistema Único de Saúde (Siscan); Sistema de Informação de Mortalidade (SIM). 


\section{Discussão}

A partir de um método de priorização de agravo de saúde pública foi possível a indicação, seleção e validação qualitativa de indicadores de desempenho que atendessem a critérios de qualidade recomendados pelo Ministério do Planejamento e a critérios específicos da área de saúde pública, de modo a torná-los viáveis à avaliação das RAS. A participação de especialistas interdisciplinares foi de suma importância para a confiabilidade e validade das decisões tomadas pelos grupos, além do alinhamento à base documental.

As metodologias qualitativas de consenso vêm constituindo, ao longo das últimas décadas, as técnicas mais apropriadas para a produção de critérios ou indicadores na área de avaliação de intervenções, especialmente na ausência de parâmetros científicos e técnicos reconhecidos e validados ${ }^{15}$. As características da TGN, como anonimato, interação e resposta estatística ao grupo contribuem para a sua difusão e aplicação na pesquisa social, em áreas como a saúde e educação ${ }^{16}$.

Em estudo realizado sobre violência doméstica, abuso sexual e exploração de crianças e adolescentes, Deslandes e colaboradores consideram que a TGN, além de ser tecnicamente recomendada, vai além de uma estratégia metodológica, expressando um princípio ético e de descoberta que valoriza as opiniões, conhecimentos e experiências dos especialistas como parceiros na produção do conhecimento ${ }^{15}$. Uma das principais vantagens dessa técnica, que se comprovou no presente estudo, foi a produção de grande número de ideias, com um maior potencial para a tomada de decisão e satisfação dos participantes, que se sentiram contemplados nas deliberações das oficinas e seus produtos, tendo em vista o processo colaborativo construído ${ }^{14,18}$.

A TGN exige a preparação prévia, que inclui pesquisa e organização de material de apoio no sentido de subsidiar oportunamente os integrantes do grupo, o que é apontado por alguns autores como uma limitação. Outra limitação diz respeito a prestar-se a apenas um único tópico, tendo em vista o reduzido tempo disponível, o que também minimiza a discussão e o pleno desenvolvimento das ideias. Por fim, sua validação científica fica comprometida por ser aplicada a um grupo pequeno de especialistas selecionados e não ter a representatividade que apresentam outras técnicas ${ }^{17}$. Outros métodos de consenso, como a técnica Delphi, compartilham das mesmas limitações, tais como a relação direta entre a confiabilidade dos resultados e o número de participantes e o número de rodadas, o efeito de fadiga dos participantes e o aumento dos custos à medida que aumenta o número de rodadas ${ }^{25}$.

Os resultados esperados com a aplicação da TGN foram alcançados com indicadores selecionados que contemplam as subdimensões de desempenho preconizadas para avaliação de sistemas de saúde, de forma adaptada às RAS. Por outro lado, foi possível qualificar a estrutura operacional da rede de atenção à saúde do câncer do colo do útero, representada no seu Centro Comunicador, Sistemas de Apoio e Pontos de Atenção Secundários e Terciários.

Os indicadores validados organizados em fichas de qualificação dentro das recomendações da RIPSA demonstram as suas características estruturantes e a organização de um sistema de monitoramento e avaliação de desempenho para a política pública relacionada ao agravo do câncer do colo do útero. A sua aplicação, contudo, é que poderá apontar para a adequação da técnica ou necessidade de reestruturação.

\section{Conclusão}

A despeito das experiências internacionais de avaliação de desempenho em sistemas de atenção integrados ${ }^{3}$, faz-se necessário o uso de modelos de avaliação adaptados ao sistema de saúde brasileiro ${ }^{7}$ e à sua situação epidemiológica, que convive com condições agudas e condições crônicas, essas últimas em ascensão ${ }^{1}$. O SUS possui diversos SIS de base nacional que captam informações ${ }^{10}$ por meio das quais é possível construir indicadores de desempenho factíveis de serem calculados e de acordo com critérios desejáveis de seleção de 
um indicador, além de representarem as diferentes subdimensões de desempenho na estrutura operacional das RAS.

O conjunto de indicadores selecionados segundo a linha de cuidado de um agravo priorizado, mediante técnica de consenso, é um aspecto positivo deste estudo por permitir uma análise do desempenho sob a perspectiva da continuidade do cuidado. A limitação, contudo, decorre da disponibilidade dos dados com abrangência para as regiões de saúde com a qualidade e atualização que se fazem necessárias.

A tecnologia da informação e a utilização de indicadores na avaliação de desempenho das RAS são elementos de apoio que possibilitam a integração entre os pontos de atenção e uma análise do atendimento às prerrogativas da integralidade da atenção com vistas à nova situação epidemiológica. Segundo Mendes, esses elementos são apontados pela Organização Mundial de Saúde (OMS) como parte das ações principais de um plano estratégico de mudança nos sistemas de atenção à saúde³ ${ }^{3}$ Esse processo implica o julgamento de valor sobre uma intervenção para racionalizar o processo decisório e a existência de informações gerenciais em saúde de qualidade que subsidiem a estruturação, cálculo e acompanhamento de indicadores de desempenho.

O monitoramento e avaliação na gestão pública necessitam estar devidamente amparados em um conjunto de indicadores que traduzam a situação do sistema de saúde ${ }^{21}$. O cálculo e a apresentação desses indicadores possibilitará a comparabilidade nas diferentes regiões de saúde do país, de forma a auxiliar os gestores na tomada de decisão no que diz respeito à adequação da rede, à infraestrutura, à qualidade da atenção, ao acesso aos serviços, à efetividade e à continuidade da assistência garantindo a integralidade da atenção. Nesse contexto, tem-se como perspectivas futuras a este estudo o cálculo e a apresentação dos indicadores validados para avaliação do desempenho da RAS do câncer do colo do útero por Região de Saúde, possibilitando assim a sua aplicação prática e a validação da metodologia de seleção de indicadores de desempenho realizada.

Espera-se que o resultado deste estudo oriente a avaliação da estrutura operacional da RAS e identifique pontos críticos que exijam maiores esforços e investimentos dos gestores no aprimoramento do sistema de atenção à saúde no tocante ao câncer de colo do útero, bem como pontos positivos que favoreçam o atendimento em saúde integral adequado à situação sanitária. A presente proposta metodológica vai além do foco avaliativo dos aspectos normativos e de governança, preenchendo uma lacuna ao contemplar aspectos relacionados à atenção no que diz respeito à resolução dos problemas de saúde da população.

\section{Referências}

1. Omram AR. The epidemiologic transition: a theory of the epidemiology of population change. Bull World Health Organ [Internet]. 2001 [cited 20150ct26];79(2):161-70. Available from: http://www.ncbi.nlm. nih.gov/pmc/articles/PMC2566347/

2. Mendes EV. As redes de atenção à saúde. Ciênc. Saúde Coletiva [Internet]. 2010 ago [citado em 2015 out 26];15(5):2297-2305. Disponível em: http://www.scielo.br/scielo.php?script=sci arttext\&pid=S141381232010000500005\&lng=en. http://dx.doi.org/10.1590/S1413-81232010000500005. Acesso em: 01 set. 2015.

3. Mendes EV. As redes de atenção à saúde. Brasília: Organização Pan-Americana da Saúde; 2011.

4. Brasil. Portaria n. 4279 de 30 de dezembro de 2010,[Internet]. Estabelece diretrizes para a organização da rede de atenção à saúde no âmbito do Sistema Único de Saúde (SUS). Diário Oficial da União. Brasília (DF), 2010 [citado em 2014 abr 26]. Disponível em URL:http://bvsms.saude.gov.br/bvs/ saudelegis/gm/2010/prt4279 3012 2010.html.

5. Brasil. Decreto n. 0 7508, de 28 de junho de 2011 [Internet].Regulamenta a Lei no 8.080, de 19 de setembro de 1990, para dispor sobre a organização do Sistema Único de Saúde - SUS, o planejamento da saúde, a assistência à saúde e a articulação interfederativa, e dá outras providências. Diário Oficial da União. Brasília (DF), 201129 jun [citado em 201529 maio 2015]. Disponível em: http://www. planalto.gov.br/ccivil 03/ ato2011-2014/2011/decreto/D7508.htm.

6. Viana ALDL, Luciana D. O processo de regionalização na saúde: contextos, condicionantes e papel das comissões Intergestores Bipartites. In: ,organizadores. Regionalização e relações federativas na política de saúde do Brasil. Rio de Janeiro: Contra Capa, 2011. p. 11-24. 
7. Viacava F, Ugá MAD, Porto S, Laguardia J, Moreira, RS. Avaliação de desempenho de sistemas de saúde: um modelo de análise. Ciênc.Saúde Coletiva [Internet], 2012 [citado em 26 out. 2015];17(4):921-34. Disponível em: http://www.scielo.br/scielo.php?script=sci arttext\&pid=S1413-81232012000400014\&lng $=$ en\&nrm=iso\&tlng $=$ pt.

8. Cecílio LCO. A epidemiologia na avaliação dos serviços de saúde: a discussão da qualidade. Saude soc. [Internet]. 1995 [citado em 26out. 2015];4(1-2):115-17. Disponível em: http://www.scielo.br/scielo. php?script=sci arttext\&pid=S0104-12901995000100024\&lng=en. http://dx.doi.org/10.1590/S010412901995000100024 .

9. Loureiro S. Sistema único de informação em saúde?integração dos dados da assistência suplementar à saúde ao sistema SUS [Internet]. In: Agência Nacional de Saúde Suplementar. Documentos técnicos de apoio ao Fórum de Saúde Suplementar de 2003, tomo 2. Rio de Janeiro, Agência Nacional de Saúde Suplementar, 2004 [citado em 26 out 2015]. Disponível em: http://saudepublica.bvs.br/pesquisa/ resource/pt/sus-18289.

10. Panitz L. Registro eletrônico de saúde e produção de informações da atenção à saúde no SUS [Dissertação]. Rio de Janeiro: Escola Nacional de Saúde Pública Sergio Arouca; 2014.

11. Porter ME, Teisberg EO. Repensando a saúde: estratégias para melhorar a qualidade e reduzir os custos. Porto Alegre: Bookman Companhia Editora; 2007.

12. Spink MJ, Menegon VM, Medrado B. Oficinas como estratégia de pesquisa: articulações teóricometodológicas e aplicações ético-políticas. Psicol Soc. 2014 [citado em 26 out 2015];26(1):32-43. Disponível em: http://www.scielo.br/pdf/psoc/v26n1/05.pdf

13. Cassiani SHB, Rodrigues LPA. Técnica de Delphi e a técnica de grupo nominal como estratégia de coleta de dados das pesquisas em Enfermagem. Acta Paul Enferm 1996 maio/jun;9(3):76-83.

14. TotikidisV. Applying the Nominal Group Technique (NGT) in community based action research for health promotion and disease prevention. Aus Commun Psychol [Internet]. 2010 [cited 26 Oct 2015];22(1):1829. Available from: https://groups.psychology.org.au/Assets/Files/Totikidis.pdf

15. Deslandes SF, Mendes CHF, Pires TO, Campos DS.Uso da Técnica Grupo Nominal e do Método Delphi para a elaboração de indicadores de avaliação das estratégias de enfrentamento da violência contra crianças e adolescentes no Brasil. Rev. Bras. Saúde Mater. Infant. [Internet] 2010 nov [citado em 2016 fev 15];10 (supl 1):s29-s37. Disponível em: http://www.scielo.br/scielo.php?script=sci arttext\&pid $=$ S1519-38292010000500003\&lng $=$ en.

16. Jones J, Hunter D. Qualitative research: consensus methods for medical and health services research. British Med J. 1995;311:376-80.

17. Potter M, Gordon S, Hamer P. The nominal group technique: a useful consensus methodology in physiotherapy research. New Zeal J Physiotherapy. 2004;32(3):126-30.

18. Gaining concensus among stakeholders through the nominal group technique [Internet]. Eval Briefs. 2006 Nov [cited 26 Oct 2015];(6):1-2. Available from: http://www.cdc.gov/healthyyouth/evaluation/pdf/brief7.pdf.

19. Fundação Oswaldo Cruz, Instituto de Comunicação e Informação em Ciência e Tecnologia. PROADESS: avaliação de desempenho do sistema de saúde brasileiro: indicadores para monitoramento:relatório final [Internet].Rio de Janeiro, 2011[cited 26 Oct 2015]. Disponível em: http://www.proadess.ícict.fiocruz.br/ SGDP-RELATORIO FINAL\%20 com sumario atualizadorev\%202014.pdf

20. Rede interagencial de informação para a saúde indicadores básicos para a saúde no Brasil: conceitos e aplicações [Internet]. 2nd ed. Brasília (DF): Organização Pan-Americana da Saúde, 2008. Disponível em: http://tabnet.datasus.gov.br/tabdata/livroidb/2ed/indicadores.pdf

21. Brasil. Ministério do Planejamento, Orçamento e Gestão. Secretaria de Planejamento e Investimentos Estratégicos. Indicadores de programas: guia metodológico [Internet]. Brasília (DF), 2010 [acesso em 26 out 2015]. Disponível em: http://www.planejamento.gov.br/secretarias/upload/Arquivos/spi/ publicacoes/100324 indicadores programas-guia metodologico.pdf.

22. Instituto Nacional do Câncer José Alencar Gomes da Silva. Painel de indicadores do câncer do colo do útero [Internet]. c2016 [citado em 26 out 2015]. Disponível em: http://www2.inca.gov.br/wps/ $\mathrm{wcm} /$ connect/acoes programas/site/home/nobrasil/programa nacional controle cancer colo utero/ indicadores/.

23. Brasil. Lei n. 12.732, de 22 de novembro de 2012 [Internet]. Dispõe sobre o primeiro tratamento de paciente com neoplasia maligna comprovada e estabelece prazo para seu início. Diário Oficial da União. 
Brasília (DF), 201223 nov [citado em 26 out 2015]. Disponível em: http://www.planalto.gov.br/ ccivil 03/ ato2011-2014/2012/lei/12732.htm

24. Instituto Nacional do Câncer José Alencar Gomes da Silva. Coordenação Geral de Prevenção e Vigilância. Divisão de Deteç̧ão Precoce e Apoio à Organização de Rede. Ficha técnica de indicadores das ações de controle do câncer do colo do útero [Internet]. 2014[citado em 26 out 2015]. Disponível em: http:// www2.inca.gov.br/wps/wcm/connect/84f26080469faa79859bed5120665fa8/FICHA+T\%C3\%89CNICA+I ndicadores+Colo+14.pdf?MOD=AJPERES\&CACHEID =84f26080469faa79859bed5120665fa8.

25. Fink A., Kosecoff J, Chassin M, Brook RH. Consensus methods: characteristics and guidelines for use [Internet]. Am J Public Health 1984 Sept, [cited 2016 Oct 26];74(9):979-83. Available from: http://www. ncbi.nlm.nih.gov/pmc/articles/PMC1651783/ 